\title{
An explicit solution to a discrete fragmentation model
}

\author{
Robert M Ziff \\ Department of Chemical Engineering, University of Michigan, Ann Arbor, MI 48109-2136, \\ USA
}

Received 26 July 1991

\begin{abstract}
The discrete binary fragmentation equation is solved explicitly for a model where the net rate of breakup of a particie of size $\bar{k}, a_{k}$, equals $(\bar{k}-1) /(\bar{k}+1)$, and the daughter-size distribution $b_{i f k}$ equals $2 /(k-1)$. This system is closely related to a model of polymer degradation considered by Simha, in which $a_{k}=1$ and $b_{i \mid k}$ is as above. In the continuum limit, both of these models go over to a continuous fragmentation model in which all particles break with an equal rate, $a(x)=1$, and the daughter-size distribution is uniform, $b(y \mid x)=2 / x$, which is at the borderline of the shattering transition.
\end{abstract}

\section{Introduction}

The general kinetic equation for discrete fragmentation is given by

$$
\frac{\mathrm{d} c_{k}(t)}{\mathrm{d} t}=-a_{k} c_{k}(t)+\sum_{j=k+1}^{\infty} a_{j} b_{k \mid j} c_{j}(t)
$$

where $c_{k}(t)$ is the concentration of $k$-size particles, or $k$-mers, at time $t, a_{k}$ is the net rate of breakup of a $k$-mer, and $b_{i \mid k}$ gives the average number of $i$-mers produced upon the breakup of a $k$-mer. Conservation of mass implies that $b_{i \mid k}$ satisfies

$$
\sum_{i=1}^{k-1} i b_{i \mid k}=k
$$

When the breakup process is binary, then $b_{i \mid k}=b_{k-i \mid k}$, and the number of particles produced in a fragmentation process is always equal two: $\sum_{i=1}^{k-1} b_{i \mid k}=2$. In this case, (1) can also be written as [1]:

$$
\frac{\mathrm{d} c_{k}(t)}{\mathrm{d} t}=-c_{k}(t) \sum_{i=1}^{k-1} F_{i, k-i}+2 \sum_{j=k+1}^{\infty} F_{k, j-k} c_{j}(t)
$$

where $F_{i j} \equiv a_{i+j} b_{i \mid i+j} / 2$ gives the net rate that $(i+j)$-mers treak into $i$-mers and $j$-meres, and is a symmetric function of $i$ and $j$.

In a continuum system, the general fragmentation equation for the evolution of the distribution $c(x, t)$ of particles of mass $x$ is given by

$$
\frac{\partial c(x, t)}{\partial t}=-a(x) c(x, t)+\int_{x}^{\infty} a(y) b(x \mid y) c(y, t) \mathrm{d} y
$$

where $b(x \mid y)$ satisfies

$$
\int_{0}^{y} x b(x \mid y) \mathrm{d} x=y .
$$


For binary breakup, $b(x \mid y)=b(y-x \mid y)$ implying $\int_{0}^{y} b(x \mid y) \mathrm{d} x=2$, and (4) may be written as

$$
\frac{\partial c(x, t)}{\partial t}=-c(x, t) \int_{0}^{x} F(y, x-y) \mathrm{d} y+2 \int_{x}^{\infty} F(x, y-x) c(y, t) \mathrm{d} y
$$

where $F(x, y) \equiv a(x+y) b(x \mid x+y) / 2$.

The fragmentation equations were first solved for the equi-reactivity model, $a_{k}=$ $k-1, b_{i \mid k}=2 /(k-1)$ (or $F_{i j}=1$ ) in the discrete form, and $a(x)=x, b(y \mid x)=2 / x$ (or $F(x, y)=1)$ in the continuous form [1,2]. These models correspond to independent random breaking of the bonds in a linear polymer system. Explicit solutions have been found for a small number of other models. Simha [1] has considered a monomerbreakoff model, in which the resulting kinetic equation is closely related to the Becker-Döring equation of nucleation kinetics (see [3] for some recent work). Bak and Bak [4] have given the solution for a continuous model where the particles break only exactly in the centre. Some other discrete fragmentation solutions are given in $[8,9]$, and explicit solutions for the continuum equation are summarized in [10-15].

The discrete fragmentation equation (1) can be solved formally by an iterative process, as shown by Simha [2], Jellinek and White [5], Baulauff and Wolf [6], and Basedow et al [7]. Using this formal solution (which we review below), it is not difficult to write computer programs to determine the $c_{k}(t)$ numerically. However, there is still interest in finding analytical solutions. These solutions allow one to understand the structure of solutions to (1), to analytically investigate scaling and asymptotic behaviour, and to investigate the violation of mass conservation. With solutions to the discrete equation, one can illustrate the transition to the continuum limit explicitly. Of course, there is also a purely mathematical interest in finding solutions to an equation such as (1).

In this paper, we consider a discrete model of fragmentation first introduced by Simha some fifty years ago [1]. While he found an explicit solution to this model, that solution involves coefficients written in terms of multiple summations that are unwieldy for large systems. Here, we make a small modification to the breakup rate in Simha's model and find an explicit solution in a compact form. In the continuum limit, both Simha's model and our model are at the borderline of the shattering transition, in which a finite fraction of the mass breaks up to zero-size particles $[13,16]$. Our solution also implies an interesting formal identity for a certain multiple summation; this identity is proven directly in appendix 1 .

\section{Simha's model}

Simha [1] considered the model defined by

$$
\begin{aligned}
& a_{k}= \begin{cases}0 & k=1 \\
1 & k>1\end{cases} \\
& b_{i \mid k}=2 /(k-1)
\end{aligned}
$$

which represents a system of binary fragmentation with $F_{i j}=1 /(i+j-1)$. Here all particles (polymers) break with equal probability, since $a_{k}=$ constant. (Contrast this to the common equi-reactivity model, where all bonds within a cluster break with equal probability.) When a particle breaks, products of all possible sizes are equally probable (as in the equi-reactivity model) since $b_{i \mid k}$ is independent of $i$. 
A general series solution of (1) for a monodisperse initial condition of particles of size $n, c_{k}(0)=\delta_{k n}$, can be found by substituting the following series into (1):

$$
c_{k}(t)= \begin{cases}\mathrm{e}^{-a_{n} t} & k=n \\ \mathrm{e}^{-a_{n} t} \sum_{i=1}^{\infty} \frac{t^{i}}{i !} B_{k i}^{(n)} & k<n\end{cases}
$$

yielding $B_{k 1}^{(n)}=a_{n} b_{k \mid n}$ and

$$
B_{k, i+1}^{(n)}=\left(a_{n}-a_{k}\right) B_{k i}^{(n)}+\sum_{j=k+1}^{n-1} a_{j} b_{k \mid j} B_{j i}^{(n)}
$$

for $i>0$. Once the solution for monodisperse initial condition is found, the solution for a general initial condition follows by linear combination. Equations (8), (9) represent one form of the general solution to (1).

For (7), this procedure yields [1]

$$
c_{k}(t)= \begin{cases}\mathrm{e}^{-t} & k=n \\ \mathrm{e}^{-t} \sum_{i=1}^{\infty} \frac{(2 t)^{i}}{i !} C_{k i}^{(n)} & k<n\end{cases}
$$

with the $C_{k i}^{(n)} \equiv 2^{-i} B_{k i}^{(n)}$ given by

$$
\begin{aligned}
C_{k 1}^{(n)} & =\frac{1}{n-1} \\
C_{k 2}^{(n)} & =\frac{1}{n-1} \sum_{j=k+1}^{n-1} \frac{1}{j-1} \\
\ldots & \\
C_{k i}^{(n)} & =\frac{1}{n-1} \sum_{j_{i}=k+1}^{n-1} \frac{1}{j_{i}-1} \ldots \sum_{j_{3}=j_{4}+1}^{n-1} \frac{1}{j_{3}-1} \sum_{j_{2}=j_{3}+1}^{n-1} \frac{1}{j_{2}-1}
\end{aligned}
$$

and $C_{k i}^{(n)}=0$ for $i>n-k$. Equations (10)-(11) are the result found by Simha, who used the notation $S_{i-1}^{(k+1)}=C_{k i}^{(n)} /(n-1)$. Simha also derived the recurrence formula (in our notation) $C_{k i}^{(n)}-C_{k-1, i}^{(n)}=-C_{k, i-1}^{(n)} /(k-1)$, wrote $C_{k 2}^{(n)}$ and $C_{k 3}^{(n)}$ in terms of the $\psi$ function, and studied the general asymptotic behaviour of the $C_{k i}^{(n)}$ for large $k$. However, the explicit evaluation of the $C_{k i}^{(n)}$ is impractical for large $n$, and no simpler expression for $c_{k}(t)$ was found.

\section{Solution of a related model}

We consider a system described by the rates

$$
a_{k}=(k-1) /(k+1) \quad b_{i \mid k}=2 /(k-1) .
$$

This is also a binary model, with $F_{i j}=1 /(i+j+1)$. The breakup products are uniformly distributed as in (7), but the rate of breakup, $a_{k}$, is weakly dependent upon $k$, approaching 1 for $k$ large, and decreasing to 0 as $k$ decreases to 1 . Models (like this

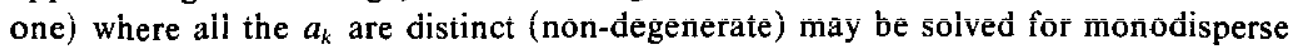
initial conditions $c_{k}(0)=\delta_{k n}$ by assuming a series solution of the form $[1,5-7]$

$$
c_{k}(t)=\sum_{i=k}^{n} A_{k i}^{(n)} \mathrm{e}^{-a_{i} t} \quad(k<i \leqslant n)
$$


where $A_{n n}^{(n)}=1$. Substituting (13) into (1), we find that the $A_{i k}^{(n)}$ satisfy the recurrence relations

$$
\begin{array}{ll}
A_{k i}^{(n)}=\frac{1}{a_{k}-a_{i}} \sum_{j=k+1}^{i} a_{j} b_{k \mid j} A_{j i}^{(n)} \quad(k<i \leqslant n) \\
A_{k k}^{(n)}=-\sum_{i=k+1}^{n} A_{k i}^{(n)} \quad(k<n) .
\end{array}
$$

The $\boldsymbol{A}_{k i}^{(n)}$ form a triangular matrix, non-zero for $k \leqslant i \leqslant n$. These recurrence formulae are applied in the following order: first $k=n-1, i=n, n-1$, then $k=n-2, i=n$, $n-1, n-2$, and so on. Equations (13)-(14) give a second form of a general solution to (1).

By programming (14) on a computer and examining the resulting $A_{k i}^{(n)}$, we have shown previously that expressions for these coefficients can often be found by inspection [9]. Carrying out this procedure for this model, we find:

$$
c_{k}(t)=\sum_{i=k}^{n}(-1)^{i-k}\left(\begin{array}{l}
i \\
k
\end{array}\right)\left(\begin{array}{l}
n \\
i
\end{array}\right) \mathrm{e}^{-(i-1) /(i+1)}
$$

Note that the binomial coefficient above can also be written $\left(\begin{array}{l}i \\ k\end{array}\right)\left(\begin{array}{l}n \\ i\end{array}\right)=\left(\begin{array}{l}n \\ k\end{array}\right)\left(\begin{array}{c}n-k \\ i-k\end{array}\right)$. Equation (15) represents a solution to (12) in a compact, explicit form. This result will be verified below.

First we show that this solution can be written in a form very similar to (10), by substituting $\mathrm{e}^{-t(i-1) /(i+1)}=\mathrm{e}^{-t} \mathrm{e}^{2 t /(i+1)}$ in (15) and expanding the second exponential of the latter expression as a power series in $(2 t)$. This procedure yields

$$
c_{k}(t)=\mathrm{e}^{-t} \sum_{i=0}^{\infty} \frac{(2 t)^{i}}{i !} H_{k i}^{(n)}
$$

where

$$
H_{k i}^{(n)}=\left(\begin{array}{l}
n \\
k
\end{array}\right) \sum_{j=k}^{n}(-1)^{j-k}\left(\begin{array}{c}
n-k \\
j-k
\end{array}\right)(j+1)^{-i}
$$

and where $H_{n 0}^{(n)}=1$, and $H_{k 0}^{(n)}=0$ for $k<n$. We have found that these $H_{k i}^{(n)}$ can also be written in a form analogous to Simha's coefficients (11);

$$
H_{k i}^{(n)}=\frac{1}{n+1} \sum_{j_{i}=k}^{n} \frac{1}{j_{i}+1} \ldots \sum_{j_{3}=j_{4}}^{n} \frac{1}{j_{3}+1} \sum_{j_{2}=j_{3}}^{n} \frac{1}{j_{2}+1} \quad H_{k 1}^{(n)}=\frac{1}{n+1} .
$$

The equivalence of (17) and (18) is proven in appendix 1 . Note that (18) does not follow directly from the series solution (8), (9). In the form of (16)-(18), the solution to our model can be seen to be very similar to Simha's solution to (7) given by (10), (11). For Simha's model, we have not been able to find a solution in a compact form similar to $(15)$.

\section{Calculation of moments}

The moments of the distribution are defined by

$$
M_{i}(t) \equiv \sum_{k=1}^{\infty} k^{i} c_{k}(t)
$$


For our model, it is convenient to first calculate the Bernoulli moments [17]:

$$
\beta_{i}(t) \equiv \sum_{k=i}^{\infty}\left(\begin{array}{l}
k \\
i
\end{array}\right) c_{k}(t)
$$

The $c_{k}$ follow from the $\beta_{i}$ simply by

$$
c_{k}(t)=\sum_{i=k}^{\infty}(-1)^{i-k}\left(\begin{array}{l}
i \\
k
\end{array}\right) \beta_{i}(t) .
$$

Comparing (21) to (15), one sees immediately that

$$
\beta_{i}=\left(\begin{array}{l}
n \\
i
\end{array}\right) \mathrm{e}^{-t(i-1) /(i+1)} \quad(0<i \leqslant n)
$$

for oụr model, and thus

$$
\begin{aligned}
& M_{1}=\beta_{1}=n \\
& M_{2}=2 \beta_{2}+\beta_{1}=n(n-1) \mathrm{e}^{-t / 3}+n \\
& M_{3}=6 \beta_{3}+6 \beta_{2}+\beta_{1}=n(n-1)(n-2) \mathrm{e}^{-t / 2}+3 n(n-1) \mathrm{e}^{-t / 3}+n .
\end{aligned}
$$

The coefficients in the relation between the $M_{i}$ and the $\beta_{i}$ are given by the Stirling numbers [17]. The zeroth moment, which gives the total number of particles, follows by summing (15):

$$
\begin{aligned}
M_{0} & =\sum_{i=1}^{n}(-1)^{i-1}\left(\begin{array}{l}
n \\
i
\end{array}\right) \mathrm{e}^{-t(i-1) /(i+1)} \\
& =\sum_{i=1}^{n}(-1)^{i-1} \beta_{i} .
\end{aligned}
$$

This result also follows directly from (21) by setting $k=0$, using that $c_{0} \equiv 0$ and $\beta_{0}=M_{0}$. As $t$ goes from 0 to $\infty$, the moments evolve from $M_{i}(0)=n^{i}$ to $M_{i}(\infty)=n$, reflecting the transformation of the system from a monodisperse distribution of size $n$ to a monodisperse distribution of unit size (the final state).

The momenents can also be derived ùsing the geñerating function $g(z, t)$ defined by

$$
\begin{aligned}
g(z, t) & =\sum_{k=1}^{\infty} z^{k} c_{k}(t) \\
& =\sum_{i=0}^{\infty}(z-1)^{i} \beta_{i}(t) .
\end{aligned}
$$

Inserting (15) into (25a), we find, after some algebra,

$$
g(z, t)=\sum_{i=1}^{n}\left(\begin{array}{l}
n \\
i
\end{array}\right)\left[(1-z)^{i}-(-1)^{i}\right] \mathrm{e}^{-t(i-1) /(i+1)} .
$$

This implies directly that the $\beta_{i}$ are given by (22) and (24), by virtue of $(25 b)$.

That the Bernoulli moments have such a simple form as (22) suggests that they might satisfy a simple closed equation. Indeed, multiplying (1), (12) by $\left(\begin{array}{l}i \\ k\end{array}\right)$ and summing over $k$ from $i$ to $n$, we find that the $\beta_{i}$ satisfy

$$
\frac{\mathrm{d} \beta_{i}}{\mathrm{~d} t}=-\frac{i-1}{i+1} \beta_{i}
$$


which implies (22), since $\beta_{i}(0)=\left(\begin{array}{l}n \\ i\end{array}\right)$. The derivation of $(27)$ is given in appendix 2 . Now, we have shown that $(22)$ in turn implies (15). Thus, $(27)$ provides a direct proof of our result (15).

\section{The continuum limit}

These results may be compared with the corresponding continuum problem, defined by $a(x)=1$ and $b(y \mid x)=2 / x$ [or $F(x, y)=1 /(x+y)]$. In this case, (4) becomes simply

$$
\frac{\partial c(x, t)}{\partial t}=-c(x, t)+2 \int_{x}^{\infty} y^{-1} c(y, t) \mathrm{d} y .
$$

Making use of a power series much like (8), one can find the solution for a monodisperse initial condition $c(x, 0)=\delta(x-v)[8,13,16]$ :

$$
c(x, t)=\mathrm{e}^{-t} \delta(x-v)+\mathrm{e}^{-t} \sum_{i=1}^{\infty} \frac{(2 t)^{i}}{i !} D_{i}(x, v)
$$

where

$$
\begin{aligned}
D_{i}(x, v) & =\frac{1}{v} \int_{x}^{v} \frac{\mathrm{d} x_{i}}{x_{i}} \ldots \int_{x_{4}}^{v} \frac{\mathrm{d} x_{3}}{x_{3}} \int_{x_{3}}^{v} \frac{\mathrm{d} x_{2}}{x_{2}} \\
& =\frac{[\ln (v / x)]^{i-1}}{v(i-1) !} .
\end{aligned}
$$

That is,

$$
c(x, t)=\mathrm{e}^{-t} \delta(x-v)+\left[\frac{2 t}{\ln (v / x)}\right]^{1 / 2} \frac{\mathrm{e}^{-t}}{v} I_{1}\left(2[2 t \ln (v / x)]^{1 / 2}\right)
$$

where $I_{1}(z)$ is the modified Bessel function. Comparing (30) with (11) and (18), it is clear that $c_{k}(t) / \varepsilon \rightarrow c(x, t)$ for both Simha's model and our model in the continuum limit $\varepsilon \rightarrow 0$, with both $x=k \varepsilon$ and $v=n \varepsilon$ fixed. The moments of this model are given simply by

$$
M_{i}(t) \equiv \int_{0}^{\infty} x^{i} c(x, t) \mathrm{d} x=v^{i} \mathrm{e}^{-t(i-1) /(i+1)}
$$

which corresponds to the leading terms of the moments for the discrete case (23).

This continuum model, in which the homogeneity $\lambda$ of $a(x)$ is equal to zero, is at the borderline of the 'shattering' [16] or 'disintegration' [13] transition. When $\lambda<0$ [11], a finite fraction of the mass of the system breaks up to particles of infinitesimal size, and the apparent mass of the system is not conserved. Thus, the models (7) and (12) are discrete analogues of a continuum model at the boundary of this transition.

\section{Conclusions}

We have presented an explicit solution to a discrete model of fragmentation, which is at the borderline of the shattering transition. This model differs from Simha's only in that the rate of breakup, $a_{k}$, rather than being constant for $k>1$, is assumed to be $(k-1) /(k+1)$. While this $a_{k}$ was chosen so that an explicit solution can be found, it 
is not physically unreasonable, as interactions between particles may be expected to decrease the breakup rate for small $k$. Mathematically, our $a_{k}$ analytically connects the necessary $a_{1}=0$ with a constant $a_{k}$ for large $k$. Our solution was verified using Bernoulli moments, and this method may be useful for other models of fragmentation as well.

\section{Acknowledgments}

This material is based upon work supported by the US National Science Foundation under Grant no DMR-86-19731.

\section{Appendix 1. Proof of the equivalence of (17) and (18)}

Clearly, from (18), the $H_{k i}^{(n)}$ satisfy the recurrence relation

$$
H_{k i}^{(n)}=\sum_{m=k}^{n} \frac{1}{m+1} H_{m, i-1}^{(n)} .
$$

To verify that (17) is equivalent to (18), we show that (17) also implies this recurrence relation:

$$
\begin{aligned}
\sum_{m=k}^{n} \frac{1}{m+1} & H_{m, i-1}^{(n)} \\
& =\sum_{m=k}^{n} \frac{1}{m+1}\left(\begin{array}{l}
n \\
m
\end{array}\right) \sum_{j=k}^{n}(-1)^{j-m}\left(\begin{array}{c}
n-m \\
j-m
\end{array}\right)(j+1)^{-i+1} \\
& =\sum_{j=k}^{n}(-1)^{j-k}\left(\begin{array}{l}
n \\
j
\end{array}\right)(j+1)^{-i+1} \sum_{m=k}^{j}(-1)^{m-k}\left(\begin{array}{c}
j \\
m
\end{array}\right) \frac{1}{m+1} \\
& =\sum_{j=k}^{n}(-1)^{j-k}\left(\begin{array}{l}
n \\
j
\end{array}\right)\left(\begin{array}{l}
j \\
k
\end{array}\right)(j+1)^{-i} \\
& =H_{k i}^{(n)}
\end{aligned}
$$

where we have used

$$
\left(\begin{array}{c}
n \\
m
\end{array}\right)\left(\begin{array}{c}
n-m \\
j-m
\end{array}\right)=\left(\begin{array}{l}
n \\
j
\end{array}\right)\left(\begin{array}{c}
j \\
m
\end{array}\right) \quad \frac{1}{m+1}\left(\begin{array}{c}
j \\
m
\end{array}\right)=\frac{1}{j+1}\left(\begin{array}{c}
j+1 \\
m+1
\end{array}\right)
$$

and the identity

$$
\sum_{m=k}^{j}(-1)^{m-k}\left(\begin{array}{c}
j+1 \\
m+1
\end{array}\right)=\left(\begin{array}{l}
j \\
k
\end{array}\right)
$$

which can be proven using a generating function. The equivalence of (17) and (18) may also be proven by showing that each gives (22) for the Bernoulli moments.

Explicitly, for $i=0,1$ and 2 , this identity reads

$$
\begin{aligned}
& \left(\begin{array}{l}
n \\
k
\end{array}\right) \sum_{j=k}^{n}(-1)^{j-k}\left(\begin{array}{l}
n-k \\
j-k
\end{array}\right)(j+1)^{-1}=\frac{1}{n+1} \\
& \left(\begin{array}{l}
n \\
k
\end{array}\right) \sum_{j=k}^{n}(-1)^{j-k}\left(\begin{array}{l}
n-k \\
j-k
\end{array}\right)(j+1)^{-2}=\frac{1}{n+1} \sum_{j=k}^{n} \frac{1}{j+1} \\
& \left(\begin{array}{l}
n \\
k
\end{array}\right) \sum_{j=k}^{n}(-1)^{j-k}\left(\begin{array}{l}
n-k \\
j-k
\end{array}\right)(j+1)^{-3}=\frac{1}{n+1} \sum_{i=k}^{n} \frac{1}{l+1} \sum_{j=l}^{n} \frac{1}{j+1} .
\end{aligned}
$$




\section{Appendix 2. Derivation of (27)}

With $a_{k}$ and $b_{i \mid k}$ given by (12), (1) becomes

$$
\frac{\mathrm{d} c_{k}}{\mathrm{~d} t}=-\frac{k-1}{k+1} c_{k}+2 \sum_{j=k+1}^{\infty} \frac{c_{j}}{j+1} .
$$

Multiplying this by $\left(\begin{array}{l}k \\ i\end{array}\right)$ and summing over $i$, we find:

$$
\begin{aligned}
\frac{\mathrm{d} \beta_{i}}{\mathrm{~d} t} & =-\sum_{k=i}^{\infty}\left(\begin{array}{l}
k \\
i
\end{array}\right) \frac{k-1}{k+1} c_{k}+2 \sum_{k=i}^{\infty}\left(\begin{array}{l}
k \\
i
\end{array}\right) \sum_{j=k+1}^{\infty} \frac{c_{j}}{j+1} \\
& =-\sum_{k=i}^{\infty}\left(\begin{array}{l}
k \\
i
\end{array}\right) \frac{k-1}{k+1} c_{k}+2 \sum_{j=i+1}^{\infty} \frac{c_{j}}{j+1} \sum_{k=i}^{j-1}\left(\begin{array}{l}
k \\
i
\end{array}\right) \\
& =-\sum_{k=i}^{\infty} c_{k}\left[\left(\begin{array}{l}
k \\
i
\end{array}\right) \frac{k-1}{k+1}-\left(\begin{array}{c}
k \\
i+1
\end{array}\right) \frac{2}{k+1}\right] \\
& =-\frac{i-1}{i+1} \sum_{k=i}^{\infty} c_{k}\left(\begin{array}{c}
k \\
i
\end{array}\right) \\
& =-\frac{i-1}{i+1} \beta_{i}
\end{aligned}
$$

where in the second line we used the identity [18]

$$
\sum_{k=i}^{j-1}\left(\begin{array}{l}
k \\
i
\end{array}\right)=\left(\begin{array}{c}
j \\
i+1
\end{array}\right) \text {. }
$$

\section{References}

[1] Simha R 1941 J. Appl. Phys. 12569

[2] Montroll E and Simha R $1940 \mathrm{~J}$. Chem. Phys. 8721

[3] Ball J M, Carr J and Penrose O 1986 Commun. Math. Phys. 104657

[4] Bak T A and Bak K 1959 Acia Chem. Scana. 131997

[5] Jellinek H H and White G 1951 J. Polymer Sci. 6745

[6] Baulauff M and Wolf G A 1984 Macromolecules 17209

[7] Basedow A M, Ebert K H and Ederer H J 1978 Macromolecules 11774

[8] Ziff R M and McGrady E D 1985 J. Phys. A: Math. Gen. 183027

[9] Ziff R M and McGrady E D 1986 Macromolecules 192513

[10] Ziff R M 1991 J. Phys. A: Math. Gen. 242821

[11] Cheng $Z$ and Redner S 1990 J. Phys. A: Math. Gen. 231233

[12] Ramkrishna D 1974 Chem. Eng. Sci. 29987

[13] Filippov A F 1961 Theory Prob. Appl. (USSR) 6275

[14] Williams M M R 1990 Aerosol Sci. and Tech. 12538

[15] Edwards B F, Cai M and Han H 1990 Phys. Rev. A 415755

[16] McGrady E D and Ziff R M 1987 Phys. Rev. Lett. 58892

[17] Riordan J 1978 An Introduction to Combinatorial Analysis (Princeton, NJ: Princeton University Press)

[18] Knuth D E 1973 The Art of Computer Programming vol 1 (Reading, MA: Addison-Wesley) ch l 